\title{
Imaging the Surface Domain Structure of Amorphous Glass-Coated Microwires by Bitter Colloid
}

\author{
K. Richter ${ }^{a, *}$, R. VARgA ${ }^{a}$, A. Thiaville ${ }^{b}$ \\ ${ }^{a}$ Institute of Physics, Faculty of Sciences, P.J. Safarik University, Park Angelinum 9, 04154 Košice, Slovakia \\ ${ }^{b}$ Laboratoire de Physique des Solides, Université Paris-Sud 11, 91400 Orsay, France
}

\begin{abstract}
The fast domain wall dynamics of amorphous glass-coated microwires can be changed drastically by properly selected annealing temperature. Here, the effect of thermal annealing on the surface domain structure of microwires is examined. Imaging the surface domain structure by Bitter colloid revealed the periodic pattern in each studied sample. Thermal annealing of microwires results in the five times increase of the domain wall velocity, as compared to the as-cast state. Influence of the surface domain structure on the fast domain wall propagation in microwires is discussed.
\end{abstract}

DOI: $10.12693 /$ APhysPolA.126.72

PACS: $75.60 . \mathrm{Ch}, 75.60 . \mathrm{Jk}$

\section{Introduction}

The domain wall dynamics of amorphous glass-coated microwires is well-known for very high domain wall velocities that reach up to $20 \mathrm{~km} / \mathrm{s} \mathrm{[1].} \mathrm{Its} \mathrm{understanding}$ attracts great attention from the technological as well as theoretical point of view. In particular, the recently developed spintronic logic devices [2] are based on the transport properties of uniaxial magnetic wires. Hence, a big attention is paid to find the mechanisms that will allow to control the domain wall velocity of a given wire.

The domain wall dynamics in thin magnetic wires was tailored by magnetic anisotropy in previous works $[3,4]$. Along with geometry of anisotropies, the domain wall dynamics can be also strongly influenced by the surface domain structure [5]. Most of recent papers on surface domain structure of microwires were devoted to the study of Co-rich samples with negative [6,7], or nearly-zero [8] coefficient of magnetostriction. However, the surface domain structure of positive-magnetostriction microwires, characterized by very high domain wall velocities, is not so clear.

Here, we observe the surface domain structure of these wires by use of Bitter colloid. It was shown in previous works [9] that thermal annealing of microwires changes the domain wall dynamics drastically. Here, the surface domain structure of as-cast samples is compared to those treated by thermal annealing. The potential influence of the surface domain structure on fast domain wall propagation is examined.

\section{Experimental}

The pieces of amorphous $\mathrm{Fe}_{77.5} \mathrm{Si}_{7.5} \mathrm{~B}_{15}$ and $\mathrm{Fe}_{49.65} \mathrm{Ni}_{27.9} \mathrm{Si}_{7.5} \mathrm{~B}_{15}$ glass-coated microwires $2 \mathrm{~cm}$

\footnotetext{
*corresponding author; e-mail: kornel.richter@gmail.com
}

in length, with metallic core of $15 \mu \mathrm{m}$ in diameter, were used for the observations. The glass-coating of microwires has been removed mechanically from the sample. Such glass-removal is usually a delicate process, because of additional mechanical stresses that can be introduced to the sample by an improper removal technique. For this reason, the glass coat was removed mechanically in several ways (by rolling and by tearing off) and many times at different pieces of sample in order to be sure that surface pattern of Bitter colloid corresponds to the surface domain structure of as-cast sample.

The remains of the glass dust were cleaned off of the metallic core surface by pressured nitrogen. A cleaned sample of microwire was placed on the glass sheet with $100 \mu \mathrm{m}$ thickness. The optimum colloid concentration was applied to the sample and $1 \mathrm{~mm}$ covering glass sheet was placed on the top of the examined wire. The metallurgical microscope supplied by D5000 Nikon photo camera was used for the observation.

\section{Results and discussion}

Figure 1 compares the Bitter colloid pattern observed at the surface of microwires with various diameters. As it is seen, the surface pattern in each sample consists of bright and dark parts that can be associated with the accumulation of magnetic particles of Bitter colloid to the position of high gradient of stray magnetic field produced by surface domain structure. Due to this fact, the high values of stray fields can be attributed to the dark parts and vice versa.

As it is seen, the sample with the largest $100 \mu \mathrm{m}$ diameter is characterized by periodic zigzag domain walls, twisting all around the wire. A similar structure has been observed previously in wires of such diameter $[10,11]$. As the microwire diameter progressively decreases, the surface domain structure becomes less complex; however, the periodic structure is maintained. This can be 
recognized in the surface domain structure of the microwire with $50 \mu \mathrm{m}$ diameter, where zigzag structure is not present. On the other hand, one may observe some kind of periodic domains, which are not inclined at the same direction (Fig. 1). Microwires of the smallest diameter $(30 \mu \mathrm{m})$ are characterized by the surface pattern consisting of dark and bright stripes inclined at a constant angle with respect to the axis of microwire. In order to correlate the surface domain structure and domain wall dynamics, two parameters of pattern were measured in as-cast and treated samples: 1 , the size of domains $d$ (measured as a distance between two bright parts) and 2, tilting angle $\alpha$ of domains (relatively to main axis of wire).
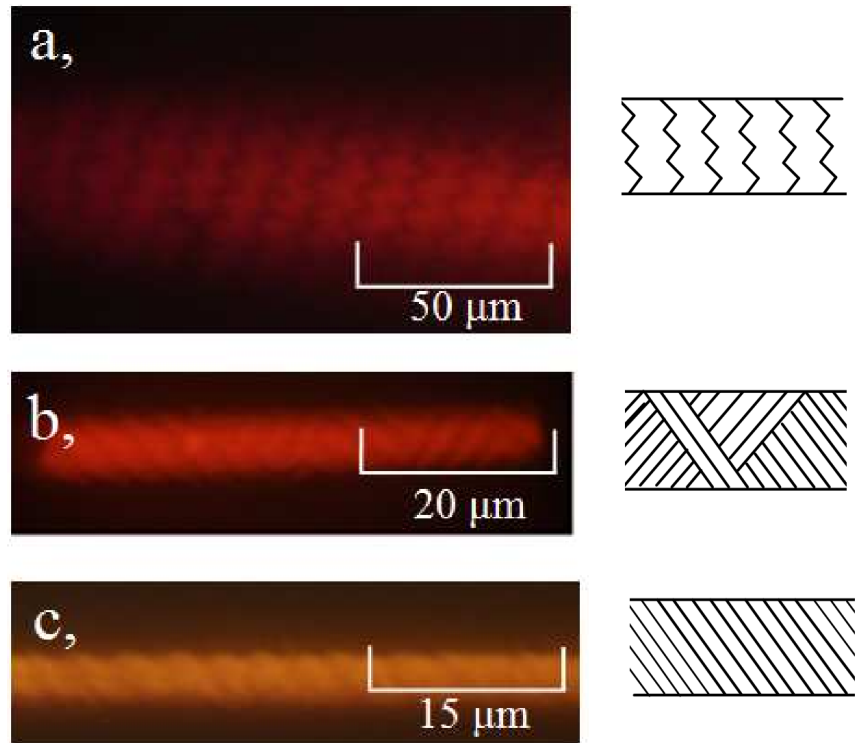

Fig. 1. Surface domain structure of microwires of various diameters (a) $125 \mu \mathrm{m}$, (b) $50 \mu \mathrm{m}$ and (c) $15 \mu \mathrm{m}$, imaged by Bitter colloid. The schematic depiction of the surface pattern is shown in the right column.

As it was shown in previous works [9], the domain wall dynamics of as-cast $\mathrm{Fe}_{77.5} \mathrm{Si}_{7.5} \mathrm{~B}_{15}$ amorphous wires is characterized by low domain wall mobility (a fiew $\left.\mathrm{m}^{2} /(\mathrm{A} \cdot \mathrm{s})\right)$ and low domain wall velocity that reach up to $1.5 \mathrm{~km} / \mathrm{s}$. Thermal annealing of the samples at $300{ }^{\circ} \mathrm{C}$ leads to drastic increase in the maximum domain wall velocity (up to $4 \mathrm{~km} / \mathrm{s}$ ), which was attributed to the faster domain wall with vortex structure. As it is seen in Tab. 1, despite the high change in domain wall dynamics, the thermal treatment of $\mathrm{Fe}_{77.5} \mathrm{Si}_{7.5} \mathrm{~B}_{15}$ was not sufficient to influence the surface domain structure. The apparent size of surface magnetic domains $(4 \mu \mathrm{m})$ is the same in both as-cast and thermally treated samples (Tab. 1).

$\mathrm{Fe}_{49.65} \mathrm{Ni}_{27.9} \mathrm{Si}_{7.5} \mathrm{~B}_{15}$ microwires are characterized by the lower magnetostriction coefficient and by the higher domain wall velocity as compared to that of FeSiB samples.

As it is seen in Tab. 1, the Bitter pattern of FeNiSiB microwire is characterized by higher apparent size of the domains (from 8 to $11 \mu \mathrm{m}$ ) as compared to FeSiB. However, the tilting angle of domains has not changed essentially (from $43^{\circ}$ to $46^{\circ}$ ).

In addition, the tilting angle of surface Bitter pattern is not strongly affected by thermal treatment as well. It proves that the change in the surface domain structure of annealed FeSiB is not the reason for high domain wall mobility observed in this material.

\section{TABLE I}

Comparison of the surface pattern obtained by Bitter colloid in two groups of microwires: (i) with low and (ii) with high value of domain wall mobility.

\begin{tabular}{c|c|c|c|c}
\hline \hline Sample & treat. & $\alpha$ & $d[\mu \mathrm{m}]$ & $S\left[\mathrm{~m}^{2} /(\mathrm{A} \mathrm{s})\right]$ \\
\hline \multirow{2}{*}{$\mathrm{Fe}_{77.5} \mathrm{Si}_{7.5} \mathrm{~B}_{15}$} & as-cast & $41^{\circ}$ & 4 & 1.6 \\
\cline { 2 - 5 } & anneal. $300^{\circ} \mathrm{C}$ & $43^{\circ}$ & 4 & 12 \\
\hline \multirow{2}{*}{$\mathrm{Fe}_{49.6} \mathrm{Ni}_{27.9} \mathrm{Si}_{7.5} \mathrm{~B}_{15}$} & as-cast & $43^{\circ}$ & 8 & 11 \\
\cline { 2 - 5 } & anneal. $300^{\circ} \mathrm{C}$ & $46^{\circ}$ & 11 & 12
\end{tabular}

An alternative explanation of these results consists in the distribution of the internal stresses that arises from the process of glass-removal.

\section{Conclusions}

The surface domain structure of microwires with (i) high and (ii) low domain wall mobility has been compared in this work. It has been shown that the effect of thermal treatment on surface domain structure is neglible.

\section{Acknowledgments}

This work was supported by the project NanoCEXmat Nr. ITMS 26220120019, Slovak VEGA Grant No. 1/0060/13, APVV-0027-11 and APVV-0266-10.

\section{References}

[1] R.Varga, A. Zhukov, V. Zhukova, J.M. Blanco, J. Gonzalez, Phys. Rev. B 76, 132406 (2007).

[2] D.A. Allwood, G. Xiong, C.C. Faulkner, D. Atkinson, D. Petit, R.P. Cowburn, Science 309, 1688 (2005).

[3] K. Richter, R. Varga, G.A. Badini-Confalonieri, M. Vázquez, Appl. Phys. Lett. 96, 182507 (2010).

[4] K. Richter, R. Varga, A. Zhukov, J. Phys.: Condens. Matter 24, 296003 (2012).

[5] Y. Nakatani, A. Thiaville, J. Miltat, Nature Materials 2, 521 (2003).

[6] A. Chizhik, A. Zhukov, J. Gonzalez, J.M. Blanco, Phys. Stat. Sol. (a) 208, 2277 (2011).

[7] M. Ipatov, A. Chizhik, V. Zhukova, J. Gonzalez, A. Zhukov, J. Appl. Phys. 109, 113924 (2011).

[8] H. Chiriac, T.-A. Óvári, M. Ţibu, J. Appl. Phys. 105, 07A310 (2009).

[9] K. Richter, R. Varga, J. Kovac, A. Zhukov, IEEE Trans. Magn. 48, 1266 (2012).

[10] M. Letcher, G.A. Jones, D.G. Lord, M. Wun-Fongle, H.T. Savage, J. Appl. Phys. 69, 5331 (1991).

[11] B. Hernando, M.L. Sánchez, V.M. Prida, J.D. Santos, J. Olivera, F.J. Belzunce, G. Badini, M. Vázquez, J. Appl. Phys. 103, 07E716 (2008). 